\title{
Recognition of Antegrade and Retrograde Atrial Activation Patterns using Hybrid Wavelet Neural Network Schemes
}

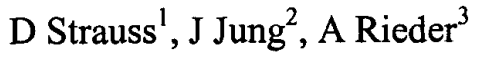 \\ ${ }^{1}$ Applied Mathematics and Computer Science, University of Mannheim, Germany \\ ${ }^{2}$ Internal Medicine III, University Hospital Homburg/Saar, Germany \\ ${ }^{3}$ Institute for Scientific Computing and Mathematical Modeling, University of Karlsruhe, Germany
}

\begin{abstract}
Currently used arrhythmia recognition algorithms in implantable cardioverter-defibrillators often fail in the discrimination of ventricular tachycardia with $1: 1$ retrograde conduction from sinus tachycardia. As new approach to solve this problem, we have developed a hybrid wavelet-neural network scheme for a recognition of antegrade atrial activation (AA) and retrograde atrial activation (RA) patterns in endocardial electrograms (EEs).

Bipolar EEs representing $A A$ and $R A$ were obtained during an electrophysiological examination. Consecutive beats within data segments of $10 \mathrm{~s}$ duration were selected. Adapted wavelet packet decompositions were applied to extract discriminating scale features in selected beats representing $A A$ and $R A$. A feed forward neural network was utilized for classifying the activation patterns based on the extracted feature vectors.

With the developed hybrid wavelet-neural network scheme a recognition of all analyzed $A A$ and $R A$ episodes was achieved.
\end{abstract}

\section{Introduction}

The implantable cardioverter-defibrillator (ICD) is accepted to be the most effective therapy for preventing sudden cardiac death due to ventricular tachycardias (VT) [1]. Despite the use of detection enhancements like rate stability or sudden onset in third generation ICD-systems, inappropriate ICD-therapy occur in up to $13 \%$ of the patients who received such a device [2]. Whether the use of the recently introduced dual-chamber ICDs [3] will contribute to a reduction of inadequate therapies is currently under investigation. A major challenge for ratealgorithms used in these devices is the discrimination of VT with 1:1 retrograde conduction from sinus tachycardia. In this case the atrial and ventricular rate is concurrent and rate independent morphological analysis techniques can be used for discrimination. Here time-domain methods based on template matching [4] or neural networks [5] are known approaches. A drawback of these methods is that the classification takes place in the original signal space where the dimensionality is often high and features being irrelevant for classification are under consideration. Recently, the superiority of wavelet decompositions before the classification over the direct application of the classifier on the original signal space was shown $[6,7]$.

We have developed a hybrid wavelet-neural network scheme for recognizing AA and RA patterns. For this, we applied adapted wavelet packet decompositions for the extraction of discriminating scale features in EEs.

\section{Methods}

\subsection{Data segments}

The data segments used in this study were obtained during a clinically indicated electrophysiological examination. A written consent was obtained from all patients. The patient population was composed as follows: One group with typical atrio-ventricular (AV) nodal tachycardia, studied as a model for patients having a spontaneous retrograde activation, and another group with clinical monomorphic VT.

In both groups, bipolar endocardial signals were obtained from the high right atrium using the distal pair of a 6-French quadripolar electrode catheter with an interelectrode spacing of $0.5 \mathrm{~cm}$ (USCI, Bard, Billerica, MA, USA). These EEs were recorded during sinus rhythm and induced or spontaneously occurring AV nodal tachycardia or during induced monomorphic VT with 1:1 retrograde conduction. The endocardial recordings were amplified (HBV 20, Biotronik, Berlin, Germany), bandpass filtered $(40-500 \mathrm{~Hz})$ and digitized at $2 \mathrm{kHz}$ with a 12 bit resolution (DT 2824-PGH, Data Translation, Marlboro, MA, USA). Data segments of 10 seconds duration were stored for subsequent analysis. 


\subsection{Feature extraction}

Consecutive beats within each recorded EE were selected by threshold application. A reliable detection threshold was defined by $27 \%$ of the largest sample amplitude of each data segment. After detection each beat was fixed within a selection window of $128 \mathrm{~ms}$. With our sampling frequency a selected beat $\boldsymbol{x}$ therefore belongs to a signal space $\mathcal{S} \subset \mathbb{R}^{K}$, where $K=256$. Wavelet-type orthonormal decompositions can be implemented via finite impulse response (FIR) normalized paraunitary (NP) two-channel filter banks [8] as building blocks in a tree-structured filter bank (as usual, we assume here the very same twochannel filter bank with a zero mean highpass filter on each decomposition level in tree). The FIR property of the filters corresponds to the application of basis functions with a compact support, a necessary condition for capturing features being compact in time.

Let $P$ and $Q$ be decomposition operators which are associated with the decimators of a two-channel NP analysis bank, $P$ : lowpass, $Q$ : highpass. With the described preprocessing of our data, the following decomposition turned out to be well suited for our feature extraction task since it discards all the irrelevant information, e.g., noise, and retains discriminating signal features:

$$
\begin{aligned}
& \left(\boldsymbol{y}_{1}, \ldots, \boldsymbol{y}_{6}\right)= \\
& \left(Q^{2} P^{2} \boldsymbol{x}, P Q P^{2} \boldsymbol{x}, Q^{2} P^{3} \boldsymbol{x}, P Q P^{3} \boldsymbol{x}, Q P^{4} \boldsymbol{x}, P^{5} \boldsymbol{x}\right)
\end{aligned}
$$

An example of the this decomposition using the well known Daubechies filters with 6 filter coefficients for the two-channel NP is shown in Fig. 1. From the decomposition we extracted a feature vector $\xi \in \mathcal{F}$ by

$$
\boldsymbol{\xi}=\left(\xi_{1}, \ldots, \xi_{6}\right):=\left(\left\|\boldsymbol{y}_{1}\right\|_{\ell^{1}}, \ldots,\left\|\boldsymbol{y}_{6}\right\|_{\ell^{1}}\right),
$$

where $\mathcal{F}$ denotes our low-dimensional feature space with $\operatorname{dim} \mathcal{F}=6$. To be independent from the overall signal energy, we applied a normalization such that $\|\boldsymbol{\xi}\|_{\ell^{1}}=1$. In the following we will use the notation $\xi^{\text {ant }}$ and $\xi^{\text {ret }}$ if the underlying pattern $x$ originates from AA and RA, respectively. Although we are able to characterize AA and RA patterns by a small feature vector, there is no guarantee that $\boldsymbol{\xi}$ represents discriminating signal features in the distinct activation patterns when using arbitrary twochannel NP building blocks in the tree. Therefore, an adaptation of these building blocks is necessary which can be realized via the lattice-structure $[9,8]$. This is an efficient implementation of two-channel FIR NP filter banks with real filter coefficients that provides a complete parameterization of orthonormal wavelet decompositions. We parameterized filters of order $N=5$ which we already used successfully for $E E$ decompositions [10, 11]. For this, a lattice structure with three distinct angles, i.e., design parameters, is necessary. It is known that the lattice angels

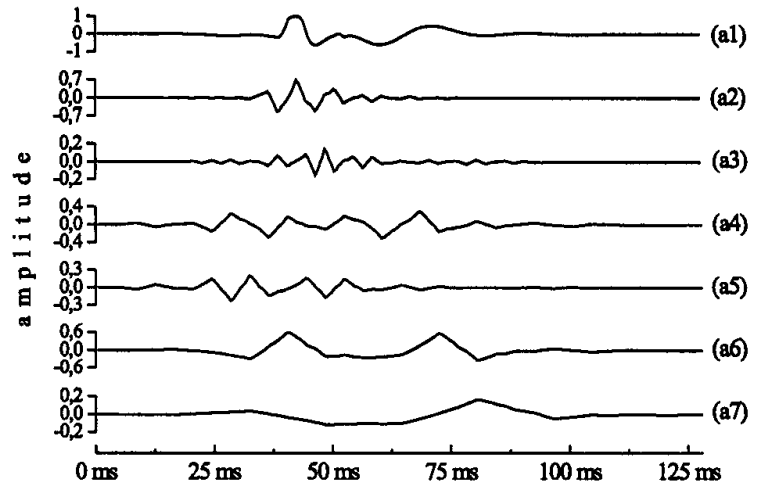

Figure 1. An exemplary decomposition using a standard filter bank: (a1) The original EE (x) and (a2)-(a7) the corresponding wavelet packet representation, i.e., the reconstructed versions of the sequences $\boldsymbol{y}_{i}, i=1, \ldots, 6$.

must add to $\pi / 4$ for implementing a highpass filter with a zero mean $[12,13]$. Fixing the last angle of the structure to $\vartheta_{2}=\pi / 4-\vartheta_{0}-\vartheta_{1}$, the angles $\vartheta=\left(\vartheta_{0}, \vartheta_{1}\right)$ are free parameters for the design of the building blocks. For the adaptation of the whole decomposition we need a discriminant information functional which measures the dissimilarity among $\boldsymbol{\xi}^{\text {ant }}(\boldsymbol{\vartheta})$ and $\boldsymbol{\xi}^{\text {ret }}(\boldsymbol{\vartheta})$ (please note that the feature vectors depend now on the angles $\vartheta$ ). For this, we applied the $J$-divergence [6] (that is a symmetric version of the more known Kullback-Leibler divergence [14]), which is defined as

$$
\begin{aligned}
J\left(\left(\boldsymbol{\xi}^{\text {ant }}(\boldsymbol{\vartheta}), \boldsymbol{\xi}^{\mathrm{ret}}(\boldsymbol{\vartheta})\right):=\right. & \sum_{n=1}^{\operatorname{dim} \mathcal{F}} \xi_{n}^{\text {ant }}(\boldsymbol{\vartheta}) \log \frac{\xi_{n}^{\text {ant }}(\boldsymbol{\vartheta})}{\xi_{n}^{\text {ret }}(\boldsymbol{\vartheta})}(3) \\
& +\sum_{n=1}^{\operatorname{dim} \mathcal{F}} \xi_{n}^{\text {ret }}(\boldsymbol{\vartheta}) \log \frac{\xi_{n}^{\text {ret }}(\boldsymbol{\vartheta})}{\xi_{n}^{\text {ant }}(\boldsymbol{\vartheta})}
\end{aligned}
$$

with the convention $\log 0=-\infty, \log (\gamma / 0)=\infty$ for $\gamma>0$, $0 \cdot( \pm \infty)=0$. The pair of angles which maximizes (3) is denoted by $\hat{\boldsymbol{\vartheta}}$.

\subsection{Classification}

The extracted feature vectors were used as input stimulus of a feed forward neural network (FFNN) with one hidden layer. This type of neural network is well accepted for classification tasks in electrocardiology, e.g., $[5,15]$.

Our FFNN consists outward of $N^{(\mathrm{I})}=\operatorname{dim} \mathcal{F}=6$ input neurons and $N^{(\mathrm{O})}=1$ output neuron. The number of neurons in the hidden layer was determined empirically. Here the restriction to $N^{(\mathrm{H})}=4$ neurons has proven to be the best compromise between FFNN complexity and performance. We used the Fermi-function, i.e., $T(\mu)=$ 
$\left(1+e^{-\mu}\right)^{-1}$ as sigmoidal transfer function of the neurons in the hidden layer.

An application of this network computes a value $\rho \in \mathbb{R}$ by

$$
\rho=\sum_{p=1}^{N^{(\mathrm{H})}} w_{p}^{(\mathrm{O})} T\left(\sum_{i=1}^{N^{(\mathrm{I})}} w_{i p}^{(\mathrm{H})} \xi_{i}(\hat{\vartheta})-\tau_{p}\right)
$$

Here $\tau$ represents the threshold vector and $w^{(0)}$ and $\boldsymbol{w}_{p}^{(\mathrm{H})}$ are the weights of the output and the hidden layer, respectively. The FFNN was trained with the backpropagation method [14] using the pre-selected sets of the five antegrade and the five retrograde activations which make up the averaged atrial activation patterns separately. The training was continued until the squared error converged to less than $1 \%$.

The FFNN was trained such that we have $\rho=0$ for AA and $\rho=1$ for RA. As decision threshold which indicates whether the underlying beat pattern originates from $A A$ and RA we used $\kappa=0.5$ for all patients. Consequently, AA is recognized if an application of the FFNN gives $\rho<\kappa$ and RA if $\rho \geq \kappa$.

To improve the reliability of the FFNN decision, we applied a so-called $X$ out of $Y$ detector which was also used in [5]. Here a final decision is only made if $X$ out of $Y$ classifications of the FFNN are the same. Our whole hybrid scheme is shown in Fig. 2.

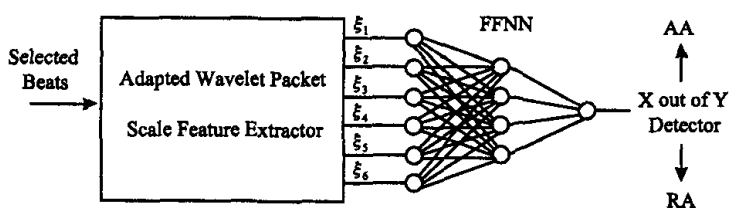

Figure 2. The whole hybrid classification scheme

\section{Results}

The decomposition example which is shown in Fig. 1 can only provide a rough survey of the morphological features which are represented on our decomposition levels $i=1, \ldots, 6$ as the representation heavily depends on the chosen wavelet packet basis. In the upper layer of Fig. 3 two averaged activation patterns are shown (AA: (a); RA: (b)). Please note, that these patterns do not differ much in their time domain representation. A similar statement can be made for the feature vectors using a non-adapted decomposition, see Fig.3(a1) and Fig.3(b1) (here we used the same Daubechies filters as above). Indeed we have a small $J$-divergence: $J\left(\boldsymbol{\xi}^{\text {ant }}\left(\boldsymbol{\vartheta}_{\mathrm{D} 6}\right), \boldsymbol{\xi}^{\text {ret }}\left(\boldsymbol{\vartheta}_{\mathrm{D} 6}\right)\right)=0.09$ (we used the natural logarithm in (3) for the numerical experiments). But the situation changes when using our adapted decomposition, see Fig.3(a2) and Fig.3(b2). Now, the feature vector are significantly dissimilar yielding a large $J$-divergence: $J\left(\xi^{\text {ant }}(\hat{\vartheta}), \xi^{\text {ret }}(\hat{\vartheta})\right)=0.60$.

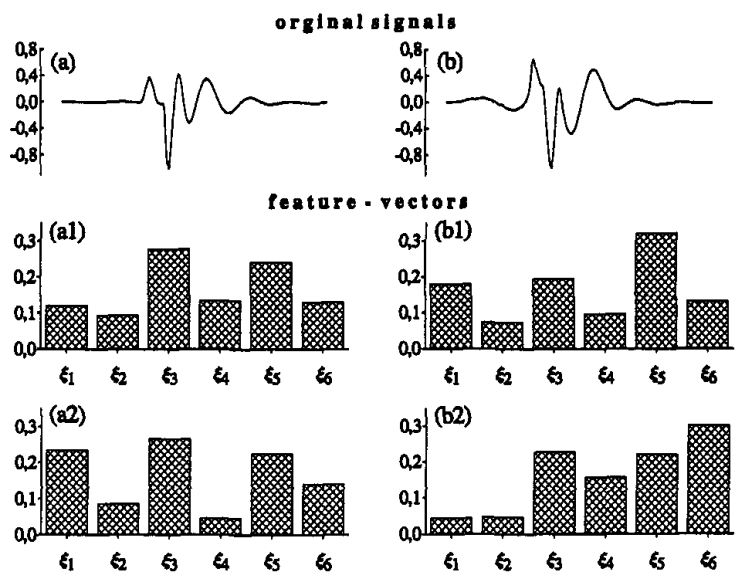

Figure 3. Extracted features in averaged activation patters: (a) antegrade activation pattern; (b) retrograde activation pattern; (a1), (b1) the extracted features using a nonadapted decomposition; (a2), (b2) the extracted features using our adapted decomposition

The whole hybrid EE classification scheme was applied to seven consecutive beats of each data segment which were not used for the adaptation and training of the FFNN, respectively. Two-hundred and fifty-four data segments (AA: 131; RA: 123) were classified by the scheme and twenty data segments were discarded by the $X$ out of $Y$ detector (we used 6 out of 7). For the classified data segments, the outputs of the FFNN are shown in Fig. 4 for all sets of $X=6$ beats underlying the classification. As noticeable, all individual atrial activations (AA: 726 beats; RA: 678 beats) were classified correctly.

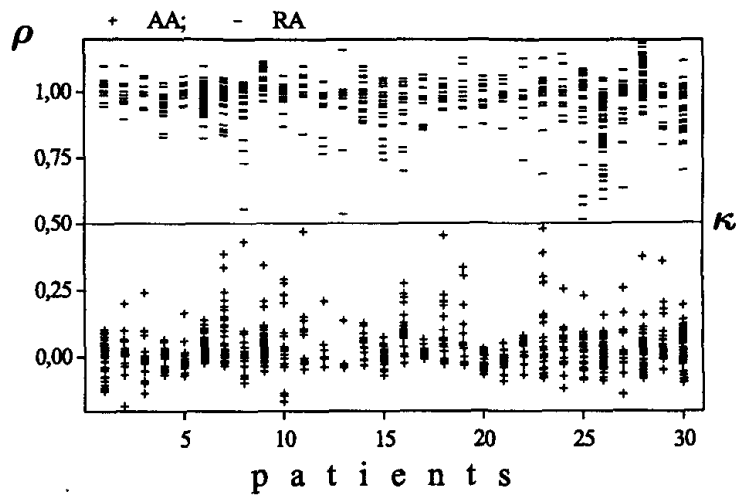

Figure 4. The results of the FFNN 


\section{Discussion and conclusion}

We have developed a hybrid wavelet-neural network scheme for a classification of AA and RA. This scheme can be used for discrimination of sinus tachycardia from VT with 1:1 retrograde conduction, a major challenge for the currently used ICD-technology. The scheme utilizes an adapted signal decomposition technique for feature extraction. This innovative approach might contribute to overcome known problems of already established time domain morphological analysis techniques since it leads to an enormous reduction of the dimensionality of the problem. In contrast to Fourier-transform domain schemes, e.g. see [15], our decomposition captures the morphological dissimilarities in the distinct activation patters since it maximizes the J-divergence between the extracted feature vectors. For the classification we used an FFNN which allows an efficient implementation and offers a high degree of generalization. Both of these properties are very desirable for our task. Note also that the efficiency of a filter bank implementation based on lattice structure is well known $[8,9,16]$. In contrast to other wavelet packet schemes, i.e, see $[6,17]$, we used a fixed architecture of the decomposition tree. In our scheme only the parameters of lattice structure must be changed for adaptation. An advantageous property for efficient real-time implementation strategies. Our hybrid scheme recognized all AA and RA episodes in the given set of data segments. Although this result is very promising, we like to emphasize that all patients were in supine position and in a resting state. Further studies are necessary to evaluate whether this method offers the same performance in an everyday clinical use, e.g., changing electrophysiological properties of the cardiac tissue over time or the presence of antiarrhythmic drugs.

\section{References}

[1] The Antiarrhythmics Versus Implantable Defibrillators (AVID) Investigators. A comparison of antiarrhythmic drug therapy with implantable defibrillators in patients resuscitated from near fatal ventricular arrhythmias. N Engl J Med 1997;337:1576-1583.

[2] Schaumann A, von zur Mühlen F, Gonska BD, Kreuzer $H$. Enhanced detection criteria in implantable cardioverterdefibrillators to avoid inappropriate therapy. Am J Cardiol 1996;78:42-50. Suppl. 5A.

[3] Nair M, Saoudi N, Kroiss D, Letac B. Automatic arrhythmia identification using analysis of the atrioventricular association. Application to a new generation of implantable defibrillators. Participating centers of the Automatic Recognition of Arrhythmia Study Group. Circulation 1997; 95:967-973.

[4] Throne RD, Jenkins JM, Winston SA, Finelli CJ, DiCarlo LA. Discrimination of retrograde from antegrade atrial activation using intracardiac electrogram waveform analysis. PACE 1989;12:1622-1630.

[5] Leong PHW, Jabri MA. Matic-An intracardiac tachycardia classification system. PACE 1992;15:1317-1331.

[6] Saito N, Coifman RR. Local discriminat bases. In: Mathematical Imaging: Wavelet Applications in Signal and Image Processing. Laine AF and Unser MA (eds.). Jul. 1994. Proc. SPIE 2303.

[7] Intrator $\mathrm{N}$, Huynh $\mathrm{Q}$, Wong $\mathrm{Y}$, Lee BHK. Wavelet feature extraction for discrimination tasks ;Proceedings of the Canadian Workshop on Information Theory. p. 83-86, Toronto, 1997.

[8] Vaidyanathan PP. Multirate Systems and Filter Banks. Englewood Cliffs, New Jersey: Prentice Hall, 1993.

[9] Vaidyanathan PP, Hoang PQ. Lattice structures for optimal design and robust implementation of two-channel perfect reconstruction qmf filter banks. IEEE Trans Acoust Speech and Signal Proc 1988;36:81-94.

[10] Jung J, Strauss D, Sinnwell T, Hohenberg H, Fries R, Wern $\mathrm{H}$, Schieffer H, Heisel A. Assessment of intersignal variability for discrimination of atrial fibrillation from atrial flutter. PACE 1998;21:2426-2430.

[11] Jung J, Strauss D, Sinnwell T, Hohenberg H, Fries R, Wern $\mathrm{H}$, Schieffer $\mathrm{H}$, Heisel A. Identification of ventricular tachycardias by means of fast wavelet analysis. In: IEEE Computers in Cardiology (25). Murray A, Swiryn S (eds.). P. 21-24, New Jersey, 1998.

[12] Strang G, Nguyen T. Wavelets and Filter Banks. Wellesley, Massachusetts: Wellesley-Cambridge Press, 1996.

[13] Delsarte PH, Macq B, Slock DTM. Signal adapted multiresolution transforms for image coding. IEEE Trans on Inform Th 1992;38:897-903.

[14] Ripley BD. Pattern Recognition and Neural Networks. New York: Cambridge University Press, 1996.

[15] Minami K, Nakajima H, Toyoshima T. Real-time discrimination of ventricular tachyarrhythmias with fouriertransform neural networks. IEEE Trans Biomed Eng 1999; 46:179-185.

[16] Kim JT, Lee YH, Isshiki T, Kunieda H. Scalable VLSI architectures for lattice-structure-based discrete wavelet transform. IEEE Trans Circuits Syst II 1998;45:1031-1043.

[17] Wickerhauser MV. Adapted Wavelet Analysis form Theory to Software. Wellesley, Massachusetts: A K Peters, Ltd., 1994.

Address for correspondence:

Daniel Strauss

Applied Mathematics and Computer Science

University of Mannheim

Building D7,27, Room 201

D-68131 Mannheim, Germany

tel./fax: ++49-621-181-2453/2477

strauss@ee.uni-sb.de 